\title{
Comparative electrophysiological effects of captopril or hydralazine combined with nitrate in patients with left ventricular dysfunction and inducible ventricular tachycardia
}

Department of
Cardiological
Sciences, St George's
Hospital Medical
School, London, UK
Y Bashir
J F Sneddon
S O'Nunain
V E Paul
S Gibson
D E Ward
A J Camm
Correspondence to
Dr Y Bashir,
Department of Cardiological
Sciences, St George's
Hospital Medical School,
Cranmer Terrace, London
SW17 0RE.
Accepted for publication
8 October 1991

Department of Cardiologica Hospital Medical School,

J F Sneddon

V E Paul

$S$ Gibson

Correspondence to

Dr Y Bashir,

Sciences, St George's

Hospital Medical School, SW17 ORE

8 October 1991

\author{
Y Bashir, J F Sneddon, S O’Nunain, V E Paul, S Gibson, D E Ward, A J Camm
}

\begin{abstract}
Objective-To assess the electrophysiological and antiarrhythmic effects of pharmacological load manipulation by an angiotensin converting enzyme (ACE) inhibitor (captopril) and a direct vasodilator (hydralazine plus isosorbide mononitrate) in patients with inducible ventricular tachycardia and impaired left ventricular function.

Design-Randomised open label crossover comparison of three regimens.

Setting-Tertiary arrhythmia referral centre.
\end{abstract}

Subjects-Eight patients with reduced left ventricular function and sustained ventricular tachycardia inducible by programmed stimulation.

Interventions-Three treatment regimens each of 48 hours duration: captopril, hydralazine plus isosorbide mononitrate, and control (no vasodilator).

Main Outcome Measures-Changes in central haemodynamics, electrophysiological parameters, and induction of ventricular tachycardia during treatment with captopril, or hydralazine combined with nitrate, compared with a control period.

Results-Both vasodilator treatments produced similar balanced reductions in peak systolic pressures and filling pressures compared with controls. Captopril had no effect on sinus cycle length, atrial refractoriness, or intraventricular conduction, but prolonged ventricular effective and functional refractory periods and QT interval during constant rate atrial pacing. Hydralazine combined with nitrate did not significantly alter any electrophysiological variable. Ventricular tachycardia was similarly inducible during all three periods.

Conclusions-Load manipulation by captopril but not hydralazine combined with nitrate prolonged ventricular refractoriness and repolarisation, possibly reflecting a combination of mechano-electrical effect with the restraining influence of ACE inhibitors on reflex sympathetic stimulation.

Numerous studies have found an increased risk of sudden death among patients with impaired left ventricular function, thought to be mediated by malignant ventricular tachyarrhythmias in most cases. ${ }^{12}$ Prevention of these life threatening arrhythmias represents an important goal, ${ }^{3}$ but the disappointing results of recent controlled trials including the Cardiac Arrhythmia Suppression Trial $(\mathrm{CAST})^{45}$ have reinforced concerns about the efficacy and safety of standard antiarrhythmic drugs in the context of reduced ventricular function. By contrast, both angiotensin converting enzyme (ACE) inhibitors and other vasodilator drugs have been shown to improve survival in patients with congestive heart failure ${ }^{67} \mathrm{With}$ growing awareness of the part played by increased myocardial wall stress and contraction-excitation feedback in the start of arrhythmias associated with left ventricular dysfunction, ${ }^{89}$ it has been suggested that these benefits of vasodilator therapy might be partly due to protective mechano-electrical effects of load reduction on susceptibility to sudden death. Inhibitors of ACE might also influence propensity to arrhythmia via indirect effects on neuroendocrine activity or sympathetic tone ${ }^{10}$ and direct effects on cardiac myocytes. ${ }^{1112}$ Although there are few data on the electrophysiological properties of these drugs in humans, experimental studies in animals have shown that vasodilators exert antiarrhythmic effects at the ventricular level, ${ }^{1314}$ and this is consistent with clinical reports of reductions in ventricular extra systole on Holter monitoring. ${ }^{15-17}$

The objective of the present study was to evaluate the influence of direct vasodilators and ACE inhibitors on the arrhythmic substrate of the failing heart. Accordingly, we compared the electrophysiological effects of a hydralazine and nitrate combination and captopril, dose titrated to achieve an equivalent degree of load manipulation, in patients with left ventricular dysfunction and ventricular tachycardia induced by programmed electrical stimulation.

\section{Patients and methods}

PATIENTS

Patients with impaired left ventricular function and recurrent ventricular tachycardia referred for serial drug testing were included in the study if they fulfilled the following criteria: (a) left ventricular ejection fraction $<40 \%$; (b) sustained ( $>30 \mathrm{~s}$ ) monomorphic ventri- 
cular tachycardia resembling the spontaneous clinical arrhythmia induced by programmed electrical stimulation; $(c)$ not previously established on an ACE inhibitor; (d) no amiodarone therapy within the preceding three months. They were excluded if they had had an acute myocardial infarction within the preceding two months or had clinical evidence of decompensated heart failure. Eight consecutive patients (six men, two women) with a mean (SD) age of 67 (6) years were recruited and Table 1 summarises their clinical features. All had coronary artery disease and a history of myocardial infarction with mean (SD) left ventricular ejection fraction of 24 (7)\%. The study protocol was approved by the district medical ethics committee and written informed consent was obtained in all cases.

\section{PROTOCOL}

As well as routine investigations, all patients underwent left ventricular and coronary angiography and a diagnostic electrophysiological study after withdrawal of antiarrhythmic and vasoactive medication for five half lives. Left ventricular ejection fraction was estimated from the right anterior oblique view using a dedicated software package based on the Dodge formula. Patients meeting the entry criteria proceeded to a randomised open label crossover study comparing electrophysiological and haemodynamic parameters during three treatment periods, each of 48 hours duration. During two limbs of the study, subjects received active treatment with either captopril or hydralazine and isosorbide mononitrate, and during the other, control, limb they received no vasodilator. Oral antiarrhythmic drugs were withheld for the duration of the protocol. Captopril or hydralazine combined with isosorbide mononitrate were administered at eight-hourly intervals (total six doses) with blood pressure monitored every four hours by sphygmomanometry, and dose adjustment aimed at achieving a $10-20 \%$ reduction in systolic blood pressure. The starting doses were $25 \mathrm{mg}$ captopril (after a test dose of $6.25 \mathrm{mg}$ ), and $50 \mathrm{mg}$ hydralazine combined with $10 \mathrm{mg}$ isosorbide mononitrate, but these were doubled if systolic pressure fell by less than $10 \%$ during the first 24 hours or halved if the fall was greater than $20 \%$. All patients underwent combined electrophysiological and haemodynamic assessment at the end of each of the three 48 hour treatment periods.

Table 1 Clinical data

\begin{tabular}{lllllll}
\hline $\begin{array}{l}\text { Patient } \\
\text { No }\end{array}$ & Age/Sex & CAD & $E F(\%)$ & $\begin{array}{l}\text { LVEDP } \\
(\mathbf{m m ~ H g})\end{array}$ & TCL (ms) & Symptoms \\
\hline 1 & $56 / \mathrm{M}$ & 2 & 28 & 12 & 240 & SCD \\
2 & $69 / \mathrm{F}$ & 1 & 15 & 30 & 280 & Syncope \\
3 & $68 / \mathrm{M}$ & 2 & 19 & 16 & 330 & Presync \\
4 & $61 / \mathrm{M}$ & 1 & 30 & 17 & 320 & Palp \\
5 & $70 / \mathrm{M}$ & 1 & 26 & 16 & 340 & Palp \\
6 & $67 / \mathrm{M}$ & 1 & 37 & 12 & 350 & Syncope \\
7 & $73 / \mathrm{M}$ & 3 & 18 & 32 & 300 & Presync \\
8 & $71 / \mathrm{F}$ & 1 & 20 & 22 & 270 & Presync \\
\hline
\end{tabular}

CAD, number of diseased coronary arteries; EF, left ventricular ejection fraction; LVEDP, left CAD, number of diseased coronary arteries; EF, left ventricular ejection fraction; LVEDP, left ventricular end diastolic pressure; TCL, cycle length of ventricular tachycardia (presenting
episode); Symptoms, symptoms during ventricular tachycardia (presenting episode); SCD, "spisode); Symptoms, symptoms during ventricular tachycardia (
ELECTROPHYSIOLOGICAL AND HAEMODYNAMIC STUDY

Patients were studied after an overnight fast and light premedication with lorazepam between 0900 and 1200 on days 3, 5, and 7 of the protocol - that is, at the end of each 48 hour treatment period. The final dose of vasodilator was given at 0600 . All catheters were inserted percutaneously using local anaesthesia with $1 \%$ lignocaine and were positioned with fluoroscopic guidance. At the first study on day 3, quadripolar electrodes were introduced via the subclavian approach to the right atrial appendage and right ventricular apex: these electrodes were retained in situ and used for electrophysiological testing at the two subsequent studies on days 5 and 7 , avoiding any change in the position of the right ventricular electrode. For haemodynamic assessment, a 7F triple lumen Swan-Ganz catheter was introduced via the femoral vein to the pulmonary artery and an $18 \mathrm{G}$ cannula was placed in the femoral artery: these catheters were removed at the end of each study and reintroduced.

After insertion of the catheters and electrodes on each occasion, patients were allowed a 15 minute rest period for stabilisation before any measurements were made. The mean and phasic arterial pressure, pulmonary artery pressure, right atrial pressure, and pulmonary capillary wedge pressure signals were recorded in held expiration with the zero reference at the mid-axillary line. Cardiac output was measured by thermodilution taking the mean of three to five consecutive measurements. The derived indices, calculated from standard equations, ${ }^{18}$ were cardiac index, systemic vascular resistance, pulmonary vascular resistance, stroke volume index, and stroke work index. All haemodynamic measurements were duplicated after 10 minutes to ensure stability $(<5 \%$ variation). The percentage reductions in peak systolic arterial and pulmonary artery pressures compared with the control period were used to assess changes in afterload.

Intracardiac and surface electrocardiographic signals were recorded at a paper speed of $100 \mathrm{~mm} / \mathrm{s}$ with a multichannel ink jet recorder (Mingograf 7, Siemens-Elema). Pacing and extrastimuli were delivered with a $2 \mathrm{~ms}$ pulse width at twice diastolic threshold using a programmable stimulator (Medtronic Model 5328). In addition to sinus cycle length, QRS duration and QT interval (lead V2) were recorded during both sinus rhythm and right atrial pacing at a cycle length of $500 \mathrm{~ms}$. Atrial and ventricular refractory periods were found by the extrastimulus method to the nearest $5 \mathrm{~ms}$ at a drive cycle length of $500 \mathrm{~ms}$. After completion of all other electrophysiological and haemodynamic measurements, programmed ventricular stimulation was performed according to a strict 12 stage protocol at the single right ventricular site: single and double extrastimuli were successively introduced during sinus rhythm and drive trains at 600,500 , and $400 \mathrm{~ms}$ cycle lengths (stages 1 to 8 ), scanning diastole with $10 \mathrm{~ms}$ increments; triple extrastimuli were then delivered during sinus rhythm and the same three drive cycles (stages 
9 to 12). Stimulation continued until the end of stage 12 or induction of sustained monomorphic ventricular tachycardia lasting for more than 30 seconds or requiring termination before then due to haemodynamic compromise. Reproducibility was checked if the arrhythmia could be terminated by pacing, but not if a DC shock was required.

If ventricular tachycardia could be induced, conventional drug testing was performed at the end of the research study. A single intravenous dose of an appropriate antiarrhythmic agent was administered and programmed ventricular stimulation repeated. Plasma drug concentrations were then checked 48 hours later just before the next study to confirm complete removal.

\section{STATISTICS}

Continuous variables are given as mean (SD) and have been compared between the three treatment periods by multiple paired $t$ tests, assuming the absence of any order or period effect. Values of $p<0.05$ were regarded as significant. Changes in the stage at which ventricular tachycardia could be induced were assessed by the sign test.

\section{Results}

Seven patients completed all three treatment periods but one patient (No 6) was withdrawn at his own request after the captopril and control limbs. The final doses of vasodilator were captopril, $12.5 \mathrm{mg} \quad(\mathrm{n}=1), 25 \mathrm{mg}$ $(\mathrm{n}=6), 50 \mathrm{mg} \quad(\mathrm{n}=1)$ and hydralazine combined with isosorbide mononitrate, $50 \mathrm{mg}$ and $10 \mathrm{mg}(\mathrm{n}=5)$ and $100 \mathrm{mg}$ and $20 \mathrm{mg}$ $(\mathbf{n}=2)$. Six patients complained of headache during hydralazine and nitrate treatment but there were no other adverse effects. Table 1 shows clinical data from the patients.

\section{HAEMODYNAMIC CHANGES}

Compared with the control period, both captopril and hydralazine combined with nitrate produced significant reductions in mean arterial and pulmonary artery pressure, with a fall in systemic vascular resistance and a rise in cardiac index (table 2). As expected, both vasodilator regimens elicited similar, balanced reductions in afterload and preload. The figure shows the mean (range) fall in peak systolic

Table 2 Changes in haemodynamic variables

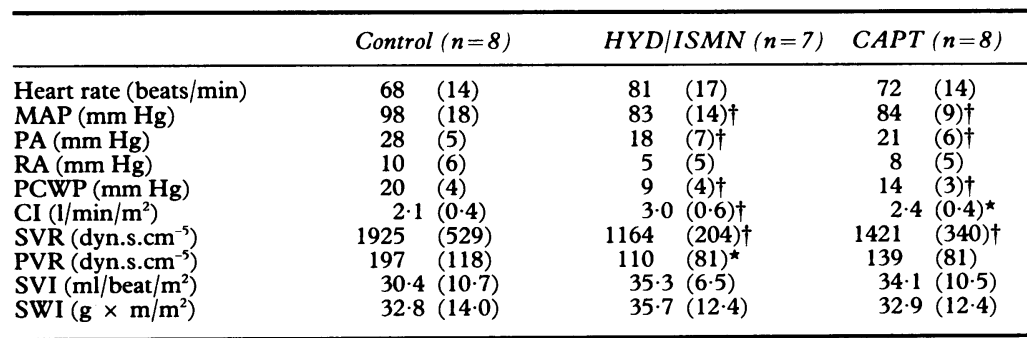

All values are mean $(\mathrm{SD}) .{ }^{\star} \mathrm{p}<0.005 v$ control; $\mathrm{tp}<0.01 v$ control. HYD, hydralazine; ISMN, isosorbide mononitrate; CAPT, captopril; MAP, mean arterial pressure; PA, mean pulmonary artery pressure; RA, mean right atrial pressure; PCWP, mean pulmonary capillary wedge pressure; CI, cardiac index; SVR, systemic vascular resistance; PVR, pulmonary vascula resistance; SVI, stroke volume index; SWI, stroke work index.

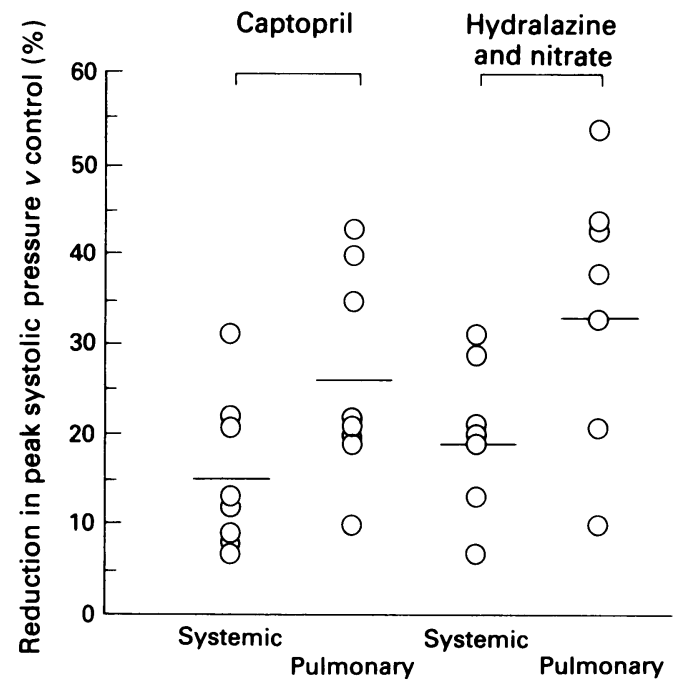

Percentage reduction in systemic and pulmonary arterial peak systolic pressure during treatment with captopril or a combination of hydralazine and nitrate compared with the control period. Horizontal bars represent mean values.

blood pressure compared with the control period. This was $15(7-30) \%$ with captopril and $19(7-31) \%$ with hydralazine and nitrate. The corresponding reductions in peak systolic pulmonary artery pressure were $26(10-43) \%$ for captopril and $33(10-54) \%$ for hydralazine and nitrate. Captopril lowered pulmonary capillary wedge pressure by $6(1-12) \mathrm{mm} \mathrm{Hg}$ and right atrial pressure by $3(-1$ to 6$) \mathrm{mm} \mathrm{Hg}$, whereas hydralazine and nitrate reduced wedge pressure by $10(5-18) \mathrm{mm} \mathrm{Hg}$ and right atrial pressure by $5(1-12) \mathrm{mm} \mathrm{Hg}$. Although the haemodynamic changes were somewhat greater with hydralazine and nitrate compared with captopril, these differences did not reach statistical significance.

ELECTROPHYSIOLOGICAL CHANGES

Table 3 summarises the electrophysiological changes. Compared with the control period, hydralazine and nitrate produced non-significant shortening of sinus cycle length, QRS duration, and uncorrected QT interval. By contrast, captopril resulted in slight but significant prolongation of mean QT interval during atrial pacing ( $500 \mathrm{~ms}$ cycle length) from 352 to $358 \mathrm{~ms}$, whereas it was unchanged during treatment with hydralazine and nitrate. Atrial refractoriness was similar during all three treatment periods, but captopril caused significant prolongation of ventricular effective and functional refractory periods compared with both the control period and treatment with hydralazine combined with nitrate.

\section{PROGRAMMED STIMULATION}

Table 4 shows the results of programmed ventricular stimulation. Ventricular tachycardia was induced in all eight patients during the control period, in seven out of eight patients during captopril treatment, and seven out of seven patients during treatment with hydralazine and nitrate. There was no significant difference in the stage at which tachycardia could be induced during any of the three limbs. 
Table 3 Mean (SD) changes in electrophysiological variables

\begin{tabular}{|c|c|c|c|c|c|c|}
\hline $\begin{array}{l}\text { Parameter } \\
\text { (ms) }\end{array}$ & $\begin{array}{l}\text { Cont } \\
(n=\end{array}$ & & $\begin{array}{l}H Y l \\
(n=\end{array}$ & 7) & $\begin{array}{l}C A \\
(n=\end{array}$ & \\
\hline $\begin{array}{l}\text { SCL } \\
\text { QRS } \\
\text { QT } \\
\text { QT }_{500} \\
\text { AERP } \\
\text { AFRP } \\
\text { VERP } \\
\text { VFRP }\end{array}$ & $\begin{array}{l}885 \\
117 \\
399 \\
351 \\
223 \\
256 \\
231 \\
265\end{array}$ & $\begin{array}{l}(265) \\
(24) \\
(61) \\
(28) \\
(35) \\
(29) \\
(26) \\
(24)\end{array}$ & $\begin{array}{l}741 \\
111 \\
379 \\
352 \\
208 \\
248 \\
228 \\
252\end{array}$ & $\begin{array}{l}(195) \\
(18) \\
(44) \\
(26) \\
(24) \\
(29) \\
(31) \\
(29)\end{array}$ & $\begin{array}{l}837 \\
121 \\
396 \\
358 \\
219 \\
256 \\
249 \\
277\end{array}$ & $\begin{array}{l}(193) \\
(24) \\
(40) \\
(25)^{\star} \\
(34) \\
(34) \\
(23) \dagger \\
(27)^{\star}\end{array}$ \\
\hline
\end{tabular}

${ }^{\star} \mathrm{p}<0.05$, captopril $v$ control; $\nmid \mathrm{p}<0.02$, captopril $v$ control. HYD, hydralazine; ISMN, isosorbide mononitrate; CAPT, captopril; SCL, sinus cycle length; QRS, QRS duration; QT, captopril; SCL, sinus cycle length; $Q R S$, $Q R S$ duration; $Q T$, QT interval during sinus rhythm; QT
atrial pacing at $500 \mathrm{~ms}$ cycle length; AERP, atrial effective atrial pacing at $500 \mathrm{~ms}$ cycle length; AERP, atrial effectio VERP, ventricular effective refractory period; VFRP ventricular functional refractory period.

Table 4 Results of programmed ventricular stimulation

\begin{tabular}{llcl}
\hline $\begin{array}{l}\text { Patient } \\
\text { No }\end{array}$ & Control & HYD/ISMN & CAPT \\
\hline 1 & 8 & 5 & 10 \\
2 & 8 & 10 & 12 \\
3 & 6 & 9 & Non-inducible \\
4 & 8 & 6 & 4 \\
5 & 4 & 4 & 4 \\
6 & 8 & Withdrew & 11 \\
7 & 2 & 2 & 2 \\
8 & 12 & 11 & 12 \\
\hline
\end{tabular}

Values represent stage of programmed stimulation protocol at which sustained ventricular tachycardia was induced (see methods).

HYD, hydralazine; ISMN, isosorbide mononitrate; CAPT, captopril.

Treatment with vasodilator had no effect on the cycle length or haemodynamic stability of induced ventricular tachycardia.

\section{Discussion}

We have shown that captopril caused an increase in ventricular functional and effective refractory periods with some prolongation of the paced QT interval, whereas an equivalent degree of load manipulation by hydralazine and nitrate resulted in no significant changes. The electrophysiological alterations produced by a vasodilator drug may represent a complex interaction of mechano-electrical effects due to reduction of myocardial wall stress, reflex changes in autonomic neural input, and direct effects on cardiac myocytes. In vitro research has shown that angiotensin II facilitates intraventricular conduction by increasing sodium current $^{11}$ and decreasing intercellular coupling resistance, ${ }^{19}$ whereas captopril directly reduces membrane conductance through $\mathrm{L}$ type calcium channels, ${ }^{12}$ but neither of these mechanisms would account for the electrophysiological changes seen with ACE inhibition in our study. Prolongation of ventricular refractoriness and repolarisation by captopril would be consistent with contraction excitation feedback, ${ }^{89}$ and a possible explanation for the lack of change with the hydralazine and nitrate combination might lie in the differing effects of these drugs on reflex autonomic response to the fall in arterial pressure. Normally, this is accompanied by a baroreceptor mediated surge in sympathetic discharge that tends to shorten cardiac action potential duration independently of heart rate and offsets any prolongation due to contraction excitation feedback. ${ }^{20}$ Adrenergic neurotransmission is, however, inhibited by ACE inhibitors. ${ }^{1021}$ This has the effect of restraining reflex sympathetic stimulation. In a comparative haemodynamic study, Daly et al showed that short-term administration of captopril to patients with congestive heart failure caused no change in net transmyocardial noradrenaline release, whereas a similar reduction in blood pressure by hydralazine and nitrate combination was accompanied by a noticeable increase in noradrenaline overflow. ${ }^{22}$ Thus it is possible that any mechanically induced change in myocardial excitability during the hydralazine and nitrate treatment period may have been masked by an opposing electrophysiological effect due to the compensatory rise in cardiac sympathetic tone. Although neurohormonal measurements were not taken, hydralazine and nitrate combination did accelerate mean heart rate from 68 to 81 beats $/ \mathrm{min}(\mathrm{p}=0.075)$, whereas there was virtually no change with captopril.

The electrophysiological effects elicited by captopril would generally be expected to exert some antiarrhythmic influence but, disappointingly, there was no significant change in the ability to induce ventricular tachycardia. Spontaneous day to day variability of ventricular tachycardia induction by programmed stimulation may, however, prevent detection of a moderate but clinically relevant antiarrhythmic effect in such a small sample of patients. $^{23}$

\section{PREVIOUS STUDIES}

There is now abundant evidence that the human myocardium exhibits the property of contraction excitation feedback. Ford and Campbell reported that afterload reduction by amyl nitrate is associated with QT interval prolongation. ${ }^{24}$ More recently, Taggart et al have showed shortening of monophasic action potential duration in response to the increase in myocardial wall stress at the end of cardiopulmonary bypass. ${ }^{25}$ This also occurs during transient aortic constriction, ${ }^{26}$ and the release phase of the Valsalva manoeuvre. ${ }^{27}$ Levine et al described similar changes in the right ventricular action potential during pulmonary balloon valvoplasty. ${ }^{28}$ All of these investigations have involved large transient load shifts and less data exist on the electrophysiological effects of sustained load manipulation by vasodilator drugs. Two recent studies have shown that administration of sodium nitroprusside to patients with impaired left ventricular function does not significantly alter ventricular refractoriness ${ }^{29}{ }^{30}$; neither study reported the effect on QT interval or any other index of repolarisation, but Carlson et al found that nitroprusside infusion caused a significant rise in plasma noradrenaline concentration. ${ }^{29}$ These results are consistent with our findings on the electrophysiological effects of hydralazine and nitrate combination and the possible confounding influence of reflex sympathetic stimulation. We are not aware of any previous human studies examining the electrophysiological properties of ACE inhibitors, but our findings generally accord with those obtained 
by de Langen et al using a closed chest pig model of chronic myocardial infarction ${ }^{13}$; they reported that angiotensin II infusion shortened ventricular refractoriness and facilitated induction of ventricular tachycardia by programmed stimulation, whereas intravenous captopril suppressed arrhythmias that were inducible in the drug free state. Holter monitoring studies in patients with chronic heart failure, furthermore, have consistently shown ACE inhibitors reduce the frequency of asymptomatic ventricular arrhythmias. ${ }^{1617}$

\section{CLINICAL IMPLICATIONS}

Sudden death accounts for 40 to $50 \%$ of the mortality in chronic heart failure and is assumed to be triggered by malignant ventricular tachyarrhythmias in a significant proportion of cases because many patients exhibit high grade ventricular extra systoles on ambulatory monitoring ${ }^{1-3}$; this viewpoint is supported by analysis of Holter electrocardiographic recordings at the time of sudden death. ${ }^{31}$ Attempts at reducing risk of sudden death with conventional antiarrhythmic drugs including amiodarone have been universally disappointing. ${ }^{3-5}$ With growing awareness of the contribution of mechano-electrical and neural mechanisms to the pathogenesis of arrhythmias in the failing heart, there are theoretical grounds for expecting ACE inhibitors to exert a protective effect, but their impact on sudden death is still a matter of dispute. It has been widely reported that the beneficial effect of enalapril in the cooperative north Scandinavian enalapril survival study (CONSENSUS) was solely due to a reduction in deaths from progressive heart failure. ${ }^{7}$ This trial, however, only recruited patients with refractory end stage heart failure, among whom sudden death accounts for a relatively low proportion of total mortality (only $22 \%$ of deaths in the placebo limb of CONSENSUS), and is more likely to be mediated by a bradyarrhythmic mechanism. ${ }^{32}$ By contrast, in the captopril multicenter research group study, only one of 53 patients on active treatment died suddenly compared with eight of 52 in the placebo group..$^{33}$ Fonarow et al have also reported a significantly lower sudden death rate among captopril treated patients compared with patients receiving hydralazine and nitrate in a randomised trial of 106 patients with chronic heart failure. ${ }^{34}$

\section{LIMITATIONS}

Practical considerations imposed several restrictions on the study, which should be borne in mind. We were only able to examine the shortterm electrophysiological effects of vasodilator therapy, but prolonged treatment with remodelling and reduction of venticular volumes might be required to assess the full impact of mechano-electrical feedback. Refractory periods were measured in the right ventricle, whereas the myocardial disease process and arrhythmic substrate are generally located in the left ventricle. Although similar reductions in peak systolic and filling pressures were achieved on the left and right sides of the heart, we cannot be sure that electrophysiological changes in the right ventricle accurately reflect the behaviour of diseased myocardium in the infarct border zone. Also changes in myocardial wall stress during the drive train preceding the extrastimulus may make it more difficult to assess the impact of pharmacologically induced load reduction on ventricular refractoriness. This problem could be avoided by using atrial pacing to stabilise the heart rate coupled to delivery of single ventricular extrastimuli. The use of oral rather than intravenous testing prevented us from assessing the effects of load manipulation on the endocardial monophasic action potential, a more sensitive and reliable index of ventricular repolarisation than the surface QT interval.

\section{CONCLUSIONS}

We showed that load manipulation by captopril but not a hydralazine and nitrate combination prolongs ventricular refractoriness and repolarisation in patients with impaired ventricular function, possibly due to the combination of mechano-electrical feedback with inhibition of reflex sympathetic stimulation. To answer some of the questions raised, we are conducting a further study with neurohormonal monitoring and intravenous drug administration to enable recording of changes in cardiac action potential and to avoid the confounding influence of day to day variation in the ability to induce ventricular arrhythmias. Despite these limitations, the results of the present study support the use of ACE inhibitors rather than direct vasodilators as one component of a pharmacological strategy to prevent sudden arrhythmic deaths associated with impaired ventricular function.

YB, JFS, and VEP are British Heart Foundation Junio Research Fellows.

1 Packer M. Sudden unexpected death in patients with congestive heart failure: a second frontier. Circulation congestive heart

2 Bigger JT. Why patients with congestive heart failure die: arrhythmias and sudden cardiac death. Circulation 1987; 75(suppl IV):28-35.

3 Francis GS. Should asymptomatic ventricular arrhythmias in patients with congestive heart failure be treated with antiarrhythmic drugs? J Am Coll Cardiol 1988;12:274-83

4 CAST Investigators. Preliminary report of the effect of flecainide and encainide on mortality in a randomized trial of arrhythmia suppression after myocardial infarction $N$ Engl J Med 1989;321:406-12.

5 Stewart RA, McKenna WJ, Poloniecki JD, Michelson JK, Das SK, Morady F, et al. Prospective randomized doubleblind placebo-controlled trial of low dose amiodarone in patients with severe heart failure and frequent ventricular ectopy [abstract]. Eur Heart J 1989;10(suppl)

6 Cohn JN, Archibald DG, Ziesche S, Franciosa JA, Harston WE, Tristani FE, et al. Effect of vasodilator therapy on mortality in chronic congestive heart failure. $N$ Engl J Med 1986;314:1547-52.

7 CONSENSUS trial study group. Effects of enalapril on mortality in severe congestive heart failure. $N \mathrm{Engl} \mathrm{J} \mathrm{Med}$ 1987;316:1429-35.

8 Lab MJ. Contraction-excitation feedback in myocardium: physiological basis and clinical relevance. Circ Res 1982 50:757-66.

9 Dean JW, Lab MJ. Arrhythmia in heart failure: role of mechanically induced changes in electrophysiology. Lancet 1989;i:1309-12.

10 Clough DP, Collis MG, Conway J, Hatton R, Keddie JR. Interaction of angiotensin-converting enzyme inhibitors with the function of the sympathetic nervous system. $\mathrm{Am}$ Cardiol 1982;49:1410-3.

11 Moorman JR, Kirsch GE, Lacerda AE, Brown AM Angiotensin II modulates cardiac sodium channels in neonatal rat. Circ Res 1989;65:1804-9.

12 Bryant SM, Ryder KO, Hart G. Effects of captopril on 
membrane current and contraction in single ventricula myocytes from guinea-pig. Br J Pharmacol 1991;102: $462-6$.

13 de Langen CDJ, de Graeff PA, van Gilst WH, Bel KJ Kingma JH, Wesseling $\mathrm{H}$. Effects of angiotensin II and captopril on inducible sustained ventricular tachycardia two weeks after myocardial infarction in the pig. two weeks after myocardial infarction

14 Dean JW, Lab MJ. Regional changes in ventricula excitability during load manipulation of the in situ pis excitability during load manipula

15 Bussman WD, Neuman K, Kaltenbach M. Effect of intravenous nitroglycerin on ventricular ectopic beats in acute myocardial infarction. Am Heart $J$ 1984;107:940-4.

16 Cleland JGF, Dargie HJ, Hodsman GP, Ball SG, Robertson JIS, Morton JJ, et al. Captopril in heart failure-a doubleblind controlled trial. Br Heart J 1984;52:530-5.

17 Webster MWI, Fitzpatrick A, Nicholls MG, Ikram H, Wells JE. Effect of enalapril on ventricular arrhythmias in congestive heart failure. Am J Cardiol 1985;56:566-9.

18 Grossman W. Cardiac catheterisation and angiography. 3rd ed. Philadelphia: Lea and Febiger, 1986:135-42.

19 Hermsmeyer K. Angiotensin II increases electrical coupling in mammalian ventricular myocardium. Cir Res 1980;47: in manm.

20 Davidowski TA, Wolf S. The QT interval during reflex cardiovascular adaptation. Circulation 1984;69:22-5.

21 McCance AJ, Forfar JC. Whole body norepinephrine kinetics in patients with chronic heart failure treated with ramipril [abstract]. J Am Coll Cardiol 1991;17:162.

22 Daly P, Rouleau JL, Cousineau D, Burgess JH, Chatterie $K$. Effects of captopril and a combination of hydralazin and isosorbide dinitrate on myocardial sympathetic tone in patients with severe congestive heart failure. Br Heart 1986;56:152-7.

23 Cooper MJ, Koo CC, Skinner MP, Mortensen PT, Hunt LJ, Richards DA, et al. Comparison of immediate versus day-to-day variability of ventricular tachycardia induction by programmed stimulation. J Am Coll Cardiol tion by programm.

24 Ford EL, Campbell NP. Effect of myocardial shortening velocity on duration of electrical and mechanical systole. Br Heart $J$ 1980;44:179-83.

25 Taggart P, Sutton PMI, Treasure T, Lab MJ, O'Brien W,
Runnalls $\mathrm{M}$, et al. Monophasic action potentials at discontinuation of cardiopulmonary bypass: evidence for contraction-excitation feedback in man. Circulation 1988; 77:1266-75.

26 Taggart P, Sutton PMI, Lab MJ, Runnalls M, O'Brien W, Treasure T. Beat-to-beat response of repolarization to transient aortic occlusion in man [abstract]. Eur Heart J 1990;11(suppl):42.

27 Taggart $P$, Sutton PMI, John R, Lab MJ, Swanton RH. aggart P, Sutton PMI, John R, Lab MJ, Swanton RH. conditions on repolarization [abstract]. Eur Heart J 1990; 11(suppl):42.

28 Levine JH, Guarnieri T, Kadish AH, White RI, Calkins H, Kan JS. Changes in myocardial repolarization in patients undergoing balloon valvuloplasty for congenital pulmonary stenosis: evidence for contraction-excitation feedback in humans. Circulation 1988;77:70-7.

29 Carlson MD, Schoenfeld MH, Garan H, Choong CY, Davidoff R, Weyman AE, et al. Programmed ventricula stimulation in patients with left ventricular dysfunction and ventricular tachycardia: effects of acute haemodynamic improvement due to nitroprusside. J Am Coll Cardiol 1989;14:1744-52.

30 Kulick DL, Bhandari AK, Hong R, Petersen R, Leon C, Rahimtoola S. Effect of acute haemodynamic decompensation on electrical inducibility of ventricular arrhythmias in patients with dilated cardiomyopathy and complex non pastained ventricular arrhythmias. Am Heart J sustained ventricu

31 de Luna AB, Coumel P, Leclercq JF. Ambulatory sudden cardiac death: mechanisms of production of fatal arrhythmia on the basis of data from 157 cases. Am Heart J 1989, 117:151-9.

32 Luu M, Stevenson WG, Stenson LW, Baron K, Warden J. Diverse mechanisms of unexpected cardiac arrests in advanced heart failure. Circulation 1989;80:1675-80.

33 Dennick LG, Maskin CS, Meyer JH, Schotz WE, Brown BW. Enalapril for congestive heart failure [letter]. $N$ Engl J Med 1987;317:1350.

34 Fonarow G, Chelimsky-Fallick C, Stevenson LW, Steven son WG, Luu M, Hamilton MA, et al. Impact of vasodilator regimen on sudden death in advanced heart failure: a randomised trial of angiotensin-convertingfailure: a randomised trial of angiotensin-convertingColl Cardiol 1991;17:92. 